\title{
Maser Emission in Astrophysical Plasmas: 2003 Robert Ellery Lecture
}

\author{
Don Melrose ${ }^{\mathrm{A}}$ \\ A School of Physics, University of Sydney, Sydney NSW 2006, Australia. \\ Email: melrose@physics.usyd.edu.au
}

Received 2004 July 21, accepted 2004 November 3

\begin{abstract}
There are three distinct types of 'coherent emission' in astrophysical plasmas: plasma emission (e.g. in solar radio bursts), electron cyclotron maser emission (e.g. in Jupiter's radio bursts), and pulsar radio emission. The development and current status of our understanding of coherent emission is reviewed, concentrating on plasma emission and electron cyclotron maser emission for which there is direct information on the distributions of electrons that produce the radiation. A generic model for a coherent emission process involves a maser generating radiation in a natural mode of the ambient plasma, and operating near marginal stability. A specific coherent emission mechanism involves the form of free energy to drive the maser, a pump that provides the free energy, and the plasma instability that leads to wave growth. The nature of coherence and its measurement through higher order intensity correlations are discussed.
\end{abstract}

Keywords: radio continuum: general — plasmas — masers — instabilities

\section{Preamble: Personal Remarks}

The award of the biennial Robert Ellery Lectureship for $2003^{1}$ cited my contributions to the field of plasma astrophysics. The topic I chose for this lecture, coherent emission processes in astrophysical plasmas, has been one of my research interests for over three decades. In an appendix I make some personal remarks on my career and my contributions to plasma astrophysics more generally.

\section{Introduction}

There are three well-recognised coherent emission processes: plasma emission, notably in solar radio bursts; electron cyclotron maser emission (ECME), in certain planetary, solar, and stellar emissions; and pulsar radio emission. Plasma emission and ECME are understood in general terms, but the identification of the pulsar emission process remains a challenge.

Radio astronomical sources may be classified as thermal, nonthermal, or coherent, depending on whether the brightness temperate is limited by the temperature in the source region, the energy (divided by Boltzmann's constant) of the nonthermal particles emitting the radiation, or whether it is higher than these limits, respectively. The observational background of the three types of coherent emission mentioned above is reviewed in Section 2, and theoretical development of our understanding of them is summarised in Section 3. An overview on the nature of coherent emission is presented in Section 4, and some more speculative ideas on the measurement of coherence are described in Section 5.

\footnotetext{
${ }^{1}$ There was no meeting of the ASA in 2003 due to the General Assembly of the IAU being held in Sydney, and the 2003 lecture was deferred to 2004.
}

\section{Historical Review}

In this section I introduce the three known coherent emission processes from an historical perspective.

\subsection{Observations of Solar Radio Bursts}

Australian radio astronomy started when the radiophysics group, concerned with radar during World War II, followed up a report by Hey (1946) of radio noise from the Sun. They used an ingenious sea-interferometric technique to localise the source of the radio bursts to sunspots (McCready, Pawsey, \& Payne-Scott 1947). The initial definitive classification of solar radio bursts into type I, II, and III was made by Wild (1950a, 1950b) and Wild \& McCready (1950) based on their appearance on dynamic spectra. The exciting agencies for type II and type III bursts were already recognised in these original papers: shock waves and streams of fast electrons, respectively. An example of a dynamic spectrum showing a type III burst is illustrated in Figure 1. The basic theoretical interpretation of the emission was recognised in this early phase of the development of the field: an exciting agent moving through the solar corona excites electron plasma oscillations (now called Langmuir waves) near the plasma frequency, $\omega_{\mathrm{p}}$, which is proportional to the square root of the electron number density, $n_{\mathrm{e}}$, and these lead to escaping radiation at the fundamental $(F)$ and second harmonic $(H)$ of $\omega_{\mathrm{p}}$. The first detailed theory was presented by Ginzburg \& Zheleznyakov (1958).

These early successes led to a systematic study of solar radio bursts, culminating in the construction of the Culgoora Radioheliograph. Initially the radioheliograph operated at $80 \mathrm{MHz}$, forming two images of the Sun per second in two circular polarisations, later extended to 


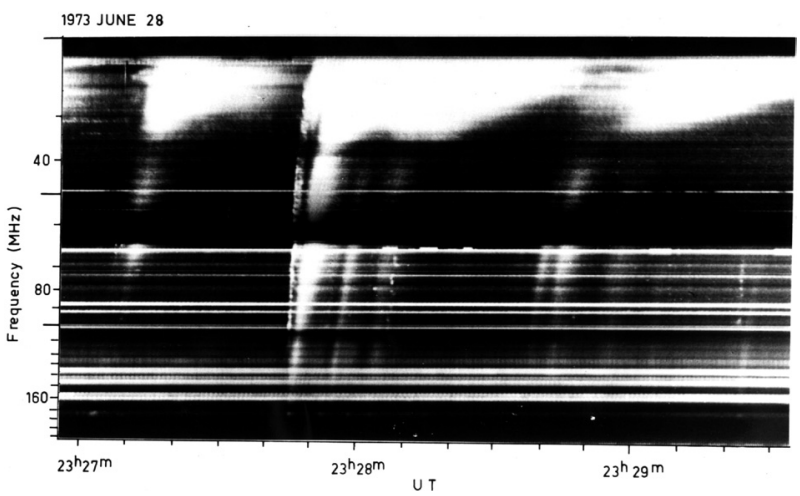

Figure 1 A dynamic spectrum showing a type III burst with both fundamental (leading trace) and harmonic (following broader trace) emission.

160, 320, and $43 \mathrm{MHz}$. The history and the scientific outcomes were summarised in the book Solar Radiophysics (McLean \& Labrum 1985).

\subsection{Properties of Solar Radio Bursts}

The general properties of solar radio bursts in the corona have been established from ground-based observations above $\sim 10 \mathrm{MHz}$. Type III bursts, and occasionally type II bursts, extend into the interplanetary medium (IPM) and have been studied by space-borne instruments at $<1 \mathrm{MHz}$ since the late 1970 s. Some notable features include the following.

Harmonic structure: Both $F$ and $H$ emission are observed in coronal type II and III bursts, but only $F$ emission is observed in type I bursts.

Brightness temperature: The maximum brightness temperature is $T_{\mathrm{B}} \sim 10^{13} \mathrm{~K}$ for type III bursts in the corona, increasing with distance in the IPM, being $\sim 10^{18} \mathrm{~K}$ near the orbit of the Earth.

Polarisation: Plasma emission is polarised in the sense of the magnetoionic o-mode; $F$ emission is usually $100 \%$ polarised in type I emission, but is never $100 \%$ polarised, and can even be unpolarised, in type II and III emission.

Simple theory can explain these features qualitatively and semiquantitatively provided that one invokes some propagation effects to cause outward ducting, scattering, and depolarisation of bursts.

\section{$2.3 D A M$ and $A K R$}

Jupiter was identified by Burke \& Franklin (1955) as a powerful source of radio emission in the decametric band (DAM). A surprising feature is that the bursts correlate with the position of the innermost Galilean satellite, Io (Biggs 1964). DAM is highly polarised in the magnetoionic $\mathrm{x}$-mode, implying opposite handedness from the north and south magnetic hemispheres. The emission frequency is close to the cyclotron frequency, $\omega_{\mathrm{B}}$, with the maximum observed frequency of $<38 \mathrm{MHz}$ corresponding to the cyclotron frequency at Jupiter's north magnetic pole. With the advent of observations from

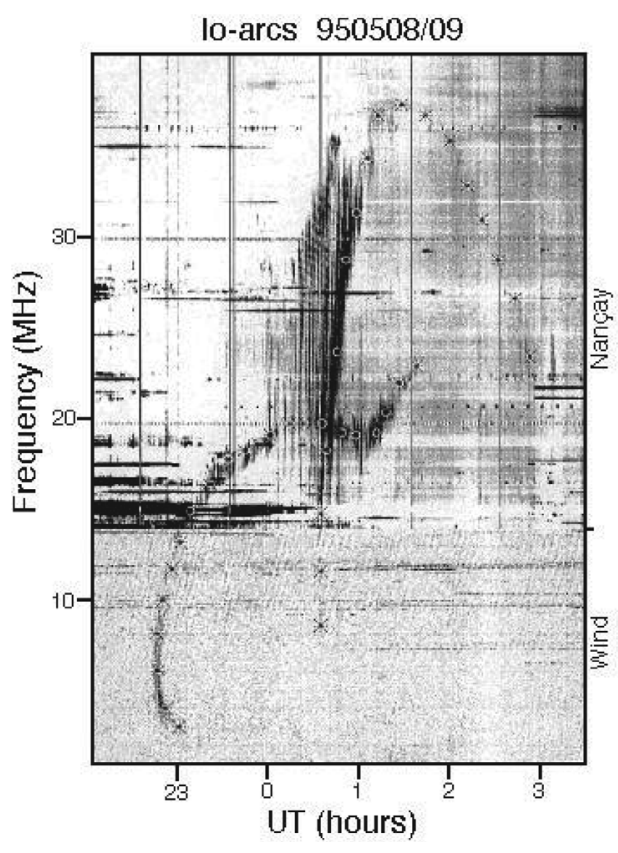

Figure 2 Io-related emission in DAM: lower half from the Wind spacecraft, and upper half from ground-based observations (Nançay). The lower arcing trace is the Io-D from the southern hemisphere, and the upper arcing trace extending to $38 \mathrm{MHz}$ is the Io-B from the northern hemisphere, and these have opposite handedness of polarisation (from Queinnec \& Zarka 1998).

space, analogous emissions were found from the Earth at $<500 \mathrm{kHz}$, called the auroral kilometric radiation (AKR), and from Saturn and Uranus. The Pioneer and Voyager flybys of Jupiter provided detailed data on the Io effect on DAM; an example is shown in Figure 2.

\subsection{Observations from Spacecraft}

One of the original motivations (pre-1970) for spacecraft radio observations was to study type III bursts at $<1 \mathrm{MHz}$. Early results showed that type III bursts extend down to $30 \mathrm{kHz}$, which corresponds to the plasma frequency in the IPM near the orbit of the Earth. The early observations were impeded by 'Earth noise' and it was some years before this was studied systematically in its own right. Two components were identified. One is AKR, at $<500 \mathrm{kHz}$, which was found to correlate with a class of 'inverted-V' precipitating electrons. AKR is closely analogous to DAM, and data on both provide strong constraints on the emission process. The other major source of Earth noise is a form of plasma emission from the Earth's bow shock, and this has analogies with both type III and type II bursts. Later spacecraft observations found analogous emissions from the bow shocks of the giant planets.

Confirmation of the theory for type III emission required that spacecraft identify not only the energetic electrons that excite type III bursts, but also confirm the presence of the Langmuir waves. The electrons were found, but for several years the Langmuir waves were elusive. It was finally realised that the Langmuir waves are present but are extremely intermittent, appearing only in highly localised, transient bursts. 
The Pioneer and Voyager spacecraft also made in situ observations inside the Jovian magnetosphere, and found $\sim 1 \mathrm{MeV}$ electrons that are accelerated near the orbit of Io. These observations provided general support for ideas proposed by Piddington \& Drake (1968) and Goldreich \& Lynden-Bell (1969) on how Io influences DAM. The idea is that the magnetic flux tube that passes through Io is frozen into Io, and hence is dragged through the Jovian magnetosphere, setting up a potential difference of order a few MV between Io and Jupiter. This potential is available to accelerate the observed electrons, but the details remain uncertain.

\subsection{Pulsar Radio Emission}

Pulsars were discovered in 1967, and over 1500 are now known. They fall into several classes, with the main two being:

Normal pulsars: $\quad$ rotation periods, $P=30 \mathrm{~ms}-10 \mathrm{~s}$; magnetic fields, $B=10^{11}-10^{13} \mathrm{G}$.

Recycled pulsars: $\quad P=1.5-30 \mathrm{~ms} ; B=10^{8}-10^{9} \mathrm{G}$.

The radio emission is extremely bright, $T_{\mathrm{B}}=10^{25}$ $10^{30} \mathrm{~K}$, compared with the brightest plasma emission and ECME, $T_{\mathrm{B}}<10^{20} \mathrm{~K}$. The emission originates from polar cap regions of rotating neutron stars. It is highly polarised, with strong linear polarisation, variable (from pulse to pulse) circular polarisation, and flips from one polarisation to the orthogonal polarisation (OPM).

A notable feature is that the radio emission is typically in the range $\sim 100 \mathrm{MHz}-10 \mathrm{GHz}$ for all pulsars. This implies that the emission process cannot be strongly dependent on either the rotation period, $P$, or the magnetic field, $B$, of the neutron star. The interpretation of the radio data is complicated by the fact that many of the observed features are determined by relativistic beaming, and the curvature of the field lines. Also, OPM suggests a separation into components in the two natural modes of the birefringent pulsar plasma, which propagate along different ray paths through the magnetosphere, further complicating the interpretation of the observations in terms of the properties at the point of emission.

The pulsar radio emission mechanism has yet to be identified unambiguously. It is likely that a single emission mechanism operates in all pulsars, and propagation effects modify the escaping radiation. Several different emission mechanisms remain under consideration, including relativistic versions of plasma emission, maser curvature emission and linear acceleration emission, free electron maser emission, and anomalous cyclotron instability. None of these has been shown capable of accounting for all the observed features of pulsar radio emission, and they are not discussed further in this paper.

\section{Coherent Emission Processes}

In this section I discuss some ideas related to coherent emission and summarise the specific instabilities involved in plasma emission and in ECME.

\subsection{Classification of Coherent Emission Mechanism}

Coherent emission mechanisms have been classified into antenna mechanisms and two classes of instabilities, referred to here as reactive and resistive.

Antenna mechanisms are based on the concept of a macrocharge: a bunch of $N$ nonthermal particles radiating in phase emits $N^{2}$ times the power per particle in incoherent emission. This requires that the bunch be smaller than a wavelength and have a small velocity spread, so that it does not disperse too quickly. The bunch must be highly localised in both coordinate space and velocity space. Such bunches cannot be set up naturally and even if one were set up, the back reaction to the emission would cause it to disperse quickly. An antenna mechanism which leads to significant emission over the large volumes and long times necessary for the observed coherent emission requires that the bunches be continually regenerated. In my opinion, this is completely unrealistic, and models based on antenna mechanisms are untenable.

Reactive mechanisms, also called hydrodynamic instabilities or overstability, involve intrinsically growing waves. Such instabilities require a distribution of particles with a small velocity spread. This requires localisation in velocity space, but there is no requirement for localisation in coordinate space. The growth rate of the instability must be greater than the Doppler width of the growing waves, so that growth occurs faster than phase mixing. The growing wave then remembers its initial phase, and a phase-coherent wave develops. The back reaction on the particles from the growing waves induces some bunching of the particles, and this self-bunching provides the positive feedback that causes the waves to grow. Growth of a reactive instability also leads to an increase in the velocity spread of the particles and hence of the Doppler width of the growing waves; eventually the increasing Doppler spread leads to phase mixing and suppression of the reactive instability. In relevant cases, the reactive instability evolves into a related maser instability, and one expects any reactive phase of growth to be only transitory.

A maser mechanism requires an inverted energy population, so that stimulated emission exceeds true absorption between the levels with inverted population. Maser emission corresponds to negative absorption. The instabilities associated with plasma emission and ECME involve inverted populations in the energy parallel and perpendicular, respectively, to the magnetic field lines. Specifically, these require a particle distribution function that satisfies

$$
\partial f\left(v_{\perp}, v_{\|}\right) / \partial v_{\|}>0, \quad \partial f\left(v_{\perp}, v_{\|}\right) / \partial v_{\perp}>0,
$$

respectively.

Whether a reactive instability or a maser instability develops depends on whether the growth rate is greater than or less than, respectively, the Doppler width of the growing waves. One expects the important instabilities in astrophysical and space plasmas to be of the maser type. 


\subsection{Coherence Volume}

The concepts of coherence and of coherent emission can be formalised in terms of the coherence volume of the radiation and the number of radiating particles in a coherence volume.

An idealised coherent wave is a sinusoidal wave of infinite duration and length, so that its frequency, $\omega$, and wave vector, $\mathbf{k}$, are well defined. A finite bandwidth, $\Delta \omega$, can be due to a variety of effects, including random changes in phase, in which case $\Delta \omega$ is determined by the rate at which the phase changes occur. Any finite bandwidth limits the coherence time to $\Delta t<2 \pi / \Delta \omega$, and hence limits the coherence length along the direction of propagation to $\Delta L<2 \pi c / \Delta \omega$. The coherence properties also depend on the angular distribution of the radiation. An angular spread $\Delta \theta$ corresponds to a coherence area $\Delta A=(2 \pi c)^{2} / \omega^{2} \pi(\Delta \theta)^{2}$. The coherence volume is the product of the coherence length and the coherence area $V_{\mathrm{c}}=\Delta L \Delta A=(2 \pi c)^{3} / \omega^{2} \Delta \omega \pi(\Delta \theta)^{2}$. More generally, the coherence volume is the inverse of the integral of $d^{3} \mathbf{k} /(2 \pi)^{3}$ over the range of $\mathbf{k}$ where the intensity is near its maximum value.

Suppose there are $\Delta n$ particles per unit volume radiating in the frequency range $\Delta \omega$ and angular range $\Delta \theta$. These particles interact coherently with the radiation, with a coherence factor $N=\Delta n V_{\mathrm{c}}$. The simple model of a macrocharge containing $N$ particles then has some validity. However, as the radiation grows, the coherence volume increases, and the simple model of a bunch needs to be used with care. One useful limit is that the maximum brightness temperature cannot exceed $N$ times the energy per particle (divided by Boltzmann's constant).

\subsection{Plasma Emission}

Plasma emission results from nonlinear processes converting Langmuir waves into escaping radiation. The maser process is the generation of the Langmuir waves, which is attributed to the bump-in-tail instability. An idealised bump-in-tail distribution is illustrated in Figure 3. In the bump-in-tail instability the motion of the particles perpendicular to the magnetic field lines is unimportant and one integrates over them, leaving a reduced distribution function that depends only on the velocity component, $v_{\|}$, along the magnetic field lines. A distribution that is an increasing function of the kinetic energy, $\frac{1}{2} m v_{\|}^{2}$, corresponds to an inverted population, and waves with phase velocity $\omega / k_{\|}$along the magnetic field grow. The backreaction, called quasilinear relaxation, to this wave growth tends to reduce the positive gradient in $v_{\|}$. An idealised example of quasilinear relaxation is illustrated in Figure 3, where the one-dimensional distribution, $F(v)$ with $v=v_{\|}$, is defined by $F\left(v_{\|}\right)=2 \pi \int_{0}^{\infty} v_{\perp} f\left(v_{\perp}, v_{\|}\right) \mathrm{d} v_{\perp}$.

The pump for this instability is simply faster particles outpacing slower particles. Suppose the energetic electrons are injected at some distant point on a magnetic field line that intersects the observer's plane. The fastest electrons arrive first at the observer's plane, and form a

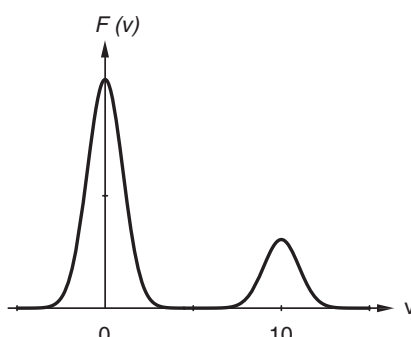

(a)

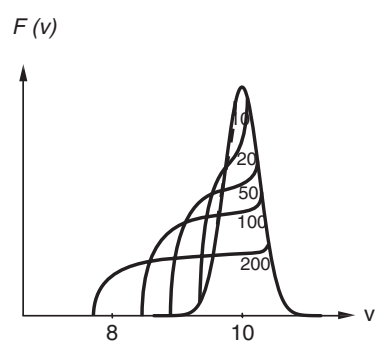

(b)
Figure 3 Evolution of bump-in-tail instability. (a) The full distribution projected onto the streaming direction, showing the thermal distribution and a displaced high energy distribution. (b) Evolution of the high-energy distribution due to quasilinear relaxation; numbers indicate time in units of the growth time (after Grognard 1985).

bump-in-tail distribution there. Any quasilinear relaxation modifies the distribution, reducing the gradient in $v_{\|}$, but the tendency of faster particles to outpace slower particles restores the positive gradient further downstream.

\subsection{ECME}

Cyclotron motion is circular motion perpendicular to a magnetic field line (plus rectilinear motion along the field line). Circular motion is simple harmonic, and in a quantum treatment it is quantised as a simple harmonic oscillator. This model suggests a perpendicular energy quantised as $\frac{1}{2} m v_{\perp}^{2}=\left(n+\frac{1}{2}\right) \hbar e B / m$, where $n$ is a simple harmonic oscillator quantum number. An inverted energy population in this case corresponds to more electrons in states with higher $n$ than with lower $n$. Higher energy states are overpopulated for $\partial f\left(v_{\perp}, v_{\|}\right) / \partial v_{\perp}>0$. Although this argument is oversimplified (the correct argument involves an intrinsically relativistic effect) it does lead to the correct conclusion concerning the essential requirement on the distribution function for ECME to develop.

A pump that can produce a distribution function with $\partial f\left(v_{\perp}, v_{\|}\right) / \partial v_{\perp}>0$ applies to electrons accelerated in a magnetic trap, that is, a dipolar-like magnetic flux loop in a planet or star. Such electrons propagating towards the surface of the planet or star are reflected due to the magnetic mirror effect. An exception is for those electrons whose initial pitch angle is sufficiently small, such that they precipitate into the dense atmosphere of the planet or star. The distribution of reflected particles then has a deficiency at small pitch angles. As illustrated in Figure 4, this implies a distribution of the required form inside the loss-cone.

An important point in understanding the interaction between electrons and radiation is the resonance condition. It is in this context that the intrinsically relativistic effect mentioned above needs to be taken into account. All the electrons that resonate with a given wave (given frequency and angle of propagation) lie on an ellipse in $v_{\perp}-v_{\|}$space. By identifying the most favourable ellipse, as illustrated in Figure 4, one can determine the frequency and angle of propagation of the fastest growing wave. 


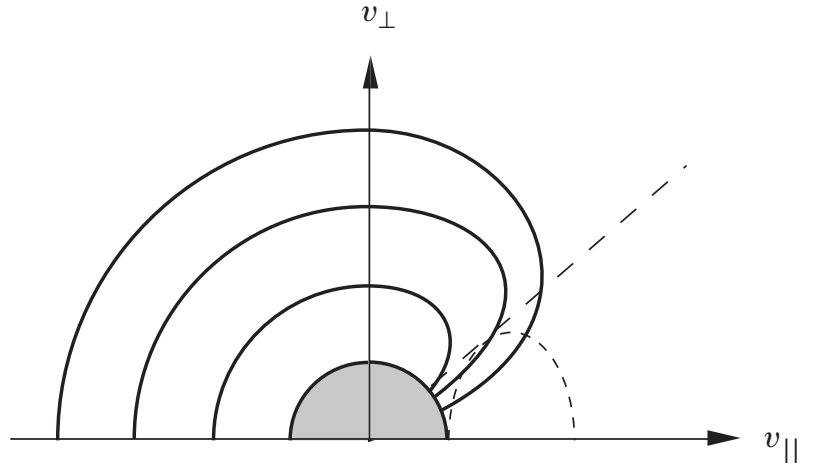

Figure 4 Loss-cone distribution: the solid lines indicate contours of the distribution function and the dashed line indicates the loss cone. All the electrons that resonate with a given wave lie along a resonant ellipse, and the ellipse that maximises the growth rate is indicated by the dashed curve.

\subsection{Marginal Stability}

One of the features of the instabilities that lead to coherent emission is that the time scales associated with the pump are very much longer than the growth time of the instability. An instability typically saturates after 10 to 30 growth times, and the time scale for the pump is much longer than this saturation time. For example, for type III bursts in the solar corona the time scales are seconds and microseconds, respectively, and for type III bursts in the IPM they are hours and seconds, respectively. This is a feature that distinguishes instabilities in astrophysical plasmas from their laboratory counterparts. The phenomena associated with instabilities in laboratory plasmas would be unobservable transient and boundary effects in astrophysical plasmas. To explain a type III burst, the instability must persist for a time very much longer than the saturation time of the instability. This is possible only if the development of the instability is very intermittent.

The concept of marginal stability is useful in understanding the implications of this difference in time scales. An average balance is achieved between the slow pump, which drives the system towards instability on a long time and a large scale, and a statistically large number of localised, transient bursts of wave growth which reduce the feature causing the wave growth. An analogy is the sand pile: a slow addition of grains of dry sand to the apex of the sand pile tends to steepen the slope of the sides of the pile, and this is opposed by localised transient slippages which reduce the slope back to a marginally stable value. The sand pile analogy is used as a basis to explain power-law distributions, such as those in solar flares and earthquakes, but the generic model does not necessarily imply powerlaw statistics for the amplitudes of the local, transient instabilities, which are typically log-normal (Robinson, Cairns, \& Gurnett 1993).

The marginal stability model has two general implications: the distribution functions should be close to marginal stability, and the large difference in the time scales for the pump and for the instability should lead to localised, transient bursts of growth. Direct measurement of the distribution functions in the source region for type III bursts in the IPM and for AKR provide support for the marginal stability model. When the one-dimensional model illustrated in Figure 3 is extended to include this effect, the measured distribution for type III electrons in the IPM is found to correspond to the self-consistent solution of the model (Grognard 1985), confirming the ideas underlying this model. As with type III electrons, measurement of the distribution function of electrons associated with AKR shows features broadly consistent with the loss-cone model, but there are also other features in the measured distributions that could lead to ECME (Louarn et al. 1990). Nearly all coherent emission is indeed bursty and intermittent. Exceptions are continua in some solar radio emission, notably the type I continuum associated with type I bursts, and these are not understood.

\section{Measurement of Coherence}

In this section observable, or potentially observable, features of coherent emission are discussed. These include direct observation of coherent structures and of the distribution functions that drive coherent emission. The statistical distribution of bursts provides a different insight into the underlying physics. Measurement of higher powers of the Stokes parameters can provide direct information on the coherence properties.

\subsection{Models for Coherence}

The defining characteristic of coherent emission is a brightness temperature, $T_{\mathrm{B}}$, higher than is consistent with any incoherent mechanism. However, this is not the only potentially observable feature of coherence. Another observable is the coherence volume, $V_{\mathrm{c}}$, which is effectively the inverse of the phase-space volume (volume of $\mathbf{k}$ space) filled by the radiation. $V_{\mathrm{c}}$ is an invariant (a Poincaré invariant) in that, like $T_{\mathrm{B}}$, it is conserved along the ray path. In particular, $k_{\mathrm{B}} T_{\mathrm{B}} / V_{\mathrm{c}}$, where $k_{\mathrm{B}}$ is Boltzmann's constant, is the energy density in the radiation, and it is also conserved along the ray path, so that its measurement provides direct information on the energy density of the radiation at the source. None of these quantities depends on the phase, which is another potentially observable feature.

Consider the phase of a wave growing through a reactive instability and through a maser instability. In the reactive case, the growth rate exceeds the bandwidth of the growing waves, implying that growth occurs faster than phase mixing. Hence, the initial phase is remembered, and a phase-coherent wave with this phase grows. A maser operates in the random phase approximation (RPA), which implies that the phase is irrelevant as far as the growth is concerned. Despite the name, the RPA does not require random phases in this context. The phase coherence of the radiation is unchanged by the growth in the RPA: input of random phase noise to a maser leads to an output of amplified random phase noise, and input of a coherent wave leads to output of an amplified coherent wave. However, 
the back reaction of wave growth in a maser does depend on the phase properties: the conventional quasilinear treatment of this backreaction assumes random phases, and the phase coherence can make this technically invalid. If individual bursts of wave growth in a maser are coherent (because the input radiation is coherent) quasilinear theory remains valid only in describing the backreaction to a large number of such coherent bursts of growth, provided their relative initial phases are random.

There is a large body of literature in quantum optics on the coherence properties of radiation, for example Klauder \& Sudarshan (1968). This theory implies the existence of potentially observable features that have been largely ignored in the astrophysical literature. This point is discussed further below in connection with moments of the intensity.

\subsection{Observations of Phase Coherent Structures}

Observations of plasma emission, ECME, and pulsar emission with sufficiently high time and frequency resolution show that some bursts contain structures with very narrow bandwidth. A notable example is the fine structures reported by Ellis (1973) in DAM. More recent observations at high time and frequency resolution appear to have resolved individual bursts of coherent emission, specifically in S-bursts in DAM (Carr \& Reyes 1999) and in emission from a pulsar (Jenet, Anderson, \& Prince 2001), compare Smits et al. (2003) however. These observations involve direct measurement of the amplitude and phase of the radiation and are technically difficult, so that it is not clear how common this feature is in coherently emitting sources.

The interpretation of such narrow band structures requires a specific model. There are analogous structures in triggered VLF emissions in the Earth's magnetosphere (Helliwell 1967). VLF emissions involve growth of whistler waves and the model of Helliwell (1967) may be adapted to apply to a phase-coherent version of ECME (Melrose 1986; Willes 2002).

\subsection{Unstable Distributions}

Direct measuring of the distribution function of the electrons exciting coherent emission has been made in the source regions for type III bursts in the IPM (Lin et al. 1981) and for AKR (Louarn et al. 1990). These observations show that the distributions are close to marginal stability, as expected. However, the time scale for measurement of the distribution function is typically much longer than the growth time, so that the distribution being measured is the relaxed distribution and not that which drives local bursts of wave growth.

\subsection{Burst Statistics}

Coherent emission is typically very bursty and the statistics of the bursts is another observable feature. The statistics may be described in terms of the probability distribution of the bursts as a function of peak intensity or of integrated (over time) intensity.

Stochastic growth (Robinson et al. 1993) has proved successful in describing the statistics associated with various forms of coherent emission. The idea is to assume that a burst, with intensity $I$, is due to localised amplification of a uniform background, intensity $I_{0}$ say, with the amplification factor, $G=I / I_{0}$, being a statistical variable. The variation of $I$ from burst to burst then determines the statistics of $G$. For an exponentially growing instability, one may write $G=e^{g}$, where $g$ is the number of $e$-folding growths. A large variety of astrophysical instabilities are consistent with log-normal statistics, which corresponds to $g$ having a Gaussian distribution (Robinson et al. 1993). This also allows one to draw conclusions concerning the relation between observed phenomena. For example, although microstructure in pulsar radiation satisfies log-normal statistics, giant pulses have a different (power-law) statistical distribution (Cairns, Johnston, \& Das 2001), indicating that they form a distinct class of microstructures.

\subsection{Moments of Stokes Parameters}

As mentioned above, there is an extensive literature on models for the coherence of radiation, and there are potentially observable properties whose importance has not been discussed widely in the astrophysical literature. Observations that could provide this additional information involve higher order correlation functions of the intensity and of the Stokes parameters.

Measurement of coherence, apart from the absolute phase, is possible through intensity correlations. This is familiar in quantum optics, where intensity correlations can be measured through photon-counting statistics. Historically it is interesting that this idea was introduced into optical physics from radio physics, notably through the Hanbury-Brown-Twiss effect, e.g., Klauder \& Sudarshan (1968). In quantum optics it is known that the rate of multiple absorption of photons depends on the coherence properties of the radiation. An idealised example is an atomic transition with frequency $\omega_{0}=N \omega$, where $\omega$ is the frequency of the laser and $N$ is an integer. The extreme case is the comparison for perfectly coherent and completely incoherent (Gaussian noise) radiation. The rate of transitions is $R_{\text {incoherent }}=N ! R_{\text {coherent }}$. In terms of intensity correlations, this corresponds to a measurement of the mean of the $N$ th power of the intensity, $\left\langle I^{N}\right\rangle$. In principle it is straightforward to build a correlator that produces not only the Stokes parameters $I, Q, U, V$, but also produces higher powers and cross-correlations between powers of these parameters. These higher powers contain additional information about the state of coherence.

It is highly desirable that both the observational feasibility and the theoretical implications of measuring the coherence properties in this way be explored. 


\section{Discussion and Conclusions}

There are three well-defined examples of coherent emission in astrophysical plasmas: plasma emission, electron cyclotron maser emission (ECME), and pulsar radio emission. These emission processes are intrinsically complicated, involving unstable systems near marginal stability. Such systems must be driven towards instability by a pump, which must operate on a long time scale and over a large volume to be observable in an astrophysical system. The pump is opposed by a statistically large number of highly localised, highly transient bursts of wave growth, which maintain the system near marginal instability. For plasma emission and ECME the respective pumps and instabilities are understood qualitatively, although numerous details are not adequately understood. For pulsar radio emission there is no consensus on which of several different proposed mechanisms is the most plausible.

One feature of the observations of all three types of coherent emission is that they include exceptional cases of very narrow frequency bursts that, while clearly related to more normal bursts, form distinct classes. The best studied of these are in Jovian S-bursts, and it seems clear that some intrinsically phase-coherent electron-wave interaction is needed to explain them (Willes 2002). For pulsars, giant bursts constitute such a distinct class (Cairns et al. 2001). For such phase-coherent growth, the maser approach emphasised in this paper is technically invalid, in that it cannot be used to describe the individual bursts of wave growth. However, it remains valid on the pump time scale, describing the effect of a statistically large number of localised bursts of phase-coherent wave growth.

Further progress in understanding coherent emission mechanisms may result from a combination of approaches: very high resolution observations, studies of burst statistics, and advances in astrophysical modelling. Currently available data on the electron distributions in situ, e.g., for type III bursts in the IPM and for AKR, are determined over times long compared with the time scale for local development of the relevant instability, and hence relate to the marginally stable distribution and not to the localised transient features that cause individual bursts of wave growth. Much higher resolution data are required to test the instability model in detail. Transient, localised bursts of wave growth result in bursty emission, and the statistics of the bursts provide information on the distribution of these transient, localised bursts of growth. For pulsar emission, uncertainties in modelling the pair plasma and in identifying the source region leave the model inadequately constrained for the pump and the relevant instability to be identified unambiguously. To a lesser extent, this also applied to suggested applications of ECME to solar and stellar bursts.

On a more speculative level, it is interesting to note that intrinsically new information on the state of coherence of radiation is available by measuring higher order correlations of the intensity (and of the other Stokes parameters). If the integration time over which the data are sampled is longer than the coherence time of the radiation, the mean $N$ th power of the intensity should be related to the $N$ th power of the mean intensity by $\left\langle I^{N}\right\rangle=N !\langle I\rangle^{N}$ (Gaussian noise), and if measurements on short time scales do not satisfy this relation then the ratio $\left\langle I^{N}\right\rangle / N !\langle I\rangle^{N}$ is a measure of an intrinsic property of the radiation related to its state of coherence.

\section{Acknowledgments}

I thank Mike Wheatland for comments on the manuscript.

\section{References}

Biggs, E. K. 1964, Natur, 203, 1008

Burke, B. F., \& Franklin, K. L. 1955, JGR, 60, 213

Cairns, I. H., Johnston, S., \& Das, P. 2001, ApJ, 561, L65

Carr, T. D., \& Reyes, F. 1999, JGR, 104, 25127

Ellis, G. R. A. 1973, PASA, 2, 191

Ginzburg, V. L., \& Zheleznyakov, V. V. 1958, SvA, 2, 653

Goldreich, P., \& Lynden-Bell, D. 1969, ApJ, 156, 59

Grognard, R. J.-M. 1985, in McLean \& Labrum (1985), p. 253

Helliwell, R. A. 1967, JGR, 72, 4773

Hey, J. S. 1946, Natur, 158, 234

Jenet, F. A., Anderson, S. B., \& Prince, T. A. 2001, ApJ, 558, 302

Klauder, J. R., \& Sudarshan, E. C. G. 1968, Fundamentals of Quantum Optics (New York: W. A. Benjamin)

Lin, R. P., Potter, D.W., Gurnett, D. A., \& Scarf, F. L. 1981, ApJ, 251,364

Louarn, P., et al. 1990, JGR, 95, 5983

McCready, L. L., Pawsey, J. L., \& Payne-Scott, P. 1947, RSPSA, 190, 357

McLean, D. J., \& Labrum, N. R. 1985, Solar Radiophysics (Cambridge: Cambridge University Press)

Melrose, D. B. 1967, P\&SS, 15, 381

Melrose, D. B. 1968, Ap\&SS, 2, 171

Melrose, D. B. 1970a, AuJPh, 23, 871

Melrose, D. B. 1970b, AuJPh, 23, 885

Melrose, D. B. 1973, AuJPh, 26, 229

Melrose, D. B. 1976, ApJ, 207, 651

Melrose, D. B. 1978, ApJ, 225, 557

Melrose, D. B. 1980, Plasma Astrophysics, Volumes 1 \& 2 (New York: Gordon \& Breach)

Melrose, D. B. 1986, JGR, 91, 7970

Melrose, D. B. 1998, Australian \& New Zealand Physicist, 35, 104

Melrose, D. B., \& Sy, W. N. 1972, AuJPh, 25, 387

Melrose, D. B., Hewitt, R. G., \& Rönnmark, K. G. 1982, JGR, 87, 5140

Piddington, J. H., \& Drake, J. F. 1968, Natur, 217, 935

Queinnec, J., \& Zarka, P. 1998, JGR, 103, 26649

Robinson, P. A., Cairns, I. H., \& Gurnett, D. A. 1993, ApJ, 407, 790

Smits, J. M., Stappers, B. W., Macquart, J.-P., Ramachandran, R., \& Kuijpers, J. 2003, A\&A, 405, 795

Twiss, R. Q. 1958, AuJPh, 11, 564

Wild, J. P. 1950a, AuJPh, 3, 399

Wild, J. P. 1950b, AuJPh, 3, 541

Wild, J. P., \& McCready, L. L. 1950, AuJPh, 3, 387

Willes, A. J. 2002, JGRA, 107, 10.1029

Wu, C. S., \& Lee, L. C. 1979, ApJ, 230, 621 


\section{Personal Remarks}

The following remarks are based in part on an earlier paper (Melrose 1998).

My interest in plasma astrophysics began in 1965. After completing a DPhil. thesis at Oxford University on an obscure problem in quantum field theory, future prospects in that field did not look promising. Australia's outstanding reputation in observational astronomy was not matched by a corresponding reputation in theoretical astrophysics, and this seemed to offer an opportunity. There was time to reflect on this during a visit back to Hobart after submitting my thesis. A specific research problem was needed to get me started in a new field, and one was identified in a discussion with Bill (G. R. A.) Ellis: how does the density of thermal plasma in the magnetosphere of Jupiter vary with height beyond the distance where the centrifugal force exceeds the gravitational force? During the following academic year at the University of Sussex my research oscillated between a particle physics problem and thinking and reading about plasma physics and astrophysics in general and Jupiter's magnetosphere in particular. One good idea (invoking an interchange instability) seemed to solve the problem concerning Jupiter's magnetosphere, and two related papers on this were accepted for publication. However, while they were in press the realisation dawned that there was a seriously embarrassing error in one of them. Both papers were withdrawn, rewritten as a single paper, and appeared as my first publication in plasma astrophysics (Melrose 1967).

An important opportunity to broaden my astrophysical interests came at the 1966 Les Houches summer school on high energy astrophysics. Of the many lecture courses at Les Houches, two turned out to be of particular importance for me, as they set the direction of my future research. The lectures were to be given by the leading Russian theoretician in cosmic ray physics, V. L. Ginzburg. However, Ginzburg was unable to attend, and the lectures were given by E. Schatzman based on Ginzburg's notes. (These had to be translated from Russian and were written up for publication by some of the participants, including me.) In these lectures was a treatment of emission and absorption processes in plasmas using a quantum mechanical formalism based on the work of V. N. Tsytovich. Tsytovich's approach appealed to me, no doubt because of our common background (shared also with Ginzburg) in quantum field theory.

My appointment at the University of Sussex was to end soon after the summer school, and the only two realistic possibilities for a position in my new field had been eliminated. Then, towards the end of the school, Al (A. G. W.) Cameron arrived to give a lecture course on nuclear astrophysics. Al had just taken up a new appointment which came with two postdoctoral positions, only one of which had been filled. After a brief interview, and a night to think it over, $\mathrm{Al}$ offered me a position to join him in New York. The agreement was for me to work on plasma astrophysics, which was not Al's field. Indeed my appointment seemed to fill one of the few gaps in his otherwise encyclopedic knowledge of astrophysics. The direction of my career had been set.

During the two years in New York with Al Cameron my research related to the scattering and acceleration of particles and the emission and absorption of waves in astrophysical plasmas. All these problems involved resonant wave-particle interactions, and one of my publications was a review-type paper that expounded my own version of the approach due to Tsytovich (Melrose 1968). Towards the end of these two years it seemed time to return to Australia. However, Al Cameron convinced me to broaden my experience by spending a further year in North America, which was spent in the Astronomy Program at the University of Maryland. A major undertaking during that year was a graduate lecture course on plasma astrophysics, for which a detailed set of lecture notes was prepared. The lecture notes were printed as a technical report, and when $\mathrm{Al}$ Cameron received a copy he arranged for a contract for them to be published. I decided to revise the notes substantially before publication. In retrospect this decision was a mistake: if the notes had been published in 1970 they would have been useful with essentially no changes. My 'revisions' ended up being a major review of the whole field, and the two-volume book Plasma Astrophysics was eventually published in 1980 (Melrose 1980).

On returning to Australia in mid-1969 (to a teaching position in Theoretical Physics at the ANU) my first major research project was to look critically at the theory of solar radio bursts. The treatment of this topic in the Maryland lectures was an uncritical discussion of the existing theory, due to Ginzburg \& Zheleznyakov (1958), which was then well over a decade old. The plasma theory in it needed to be updated, and I did this in two papers (Melrose 1970a, 1970b), and then, with my first PhD student, generalised the theory to include a magnetic field (Melrose \& Sy 1972). Although aware that the solar radiophysics group at the CSIRO Division of Radiophysics was a world leader in the field, there had been no contact between us. My first encounter with this group was at an Astronomical Society of Australia meeting in mid-1971. After he had given a talk, I asked Steve (S. F.) Smerd, who was the theoretician in the group then led by Paul (J. P.) Wild, a question, and he clearly had not remembered me from a brief meeting at a COSPAR meeting in Hobart in 1965, and in reply to my question, he suggested that I 'ask Melrose about that because he is the expert.' Thus began what turned out to be a fruitful collaboration, initially with the solar group in the Division of Radiophysics, and more widely within the Division after a six-month visit in 1973. Steve Smerd died unexpectedly a few days before my transfer to a chair at the University of Sydney in January 1979.

Early cyclotron theories for DAM were based on treating coherent emission in terms of bunches, which I regarded as unacceptable. I proposed a version of the theory of ECME (Melrose 1973) based on a theory for cyclotron instability in laboratory plasmas. This theory was later applied in detail to DAM and to AKR (Melrose 
1976). However, this version neglected an important relativistic effect, which was included by Wu \& Lee (1979). This was an opportunity missed by me, as Jim (J. A.) Roberts had pointed out the essential nature of this relativistic effect in the much earlier theory of ECME due to Twiss (1958). This relativistic effect is included explicitly in the concept of a resonance ellipse (Melrose, Hewitt, \& Rönnmark 1982), as mentioned in connection with Figure 4.

My interest in pulsar radio emission was initially motivated by it being a further example of coherent emission for which the theory was also based on emission by bunches. I proposed an alternative emission process (Melrose 1978). The interpretation of pulsar radio emission remains one of my current research interests.

It is interesting for me to reflect on what gave me my initial successes in plasma astrophysics. This was neither the influence of a mentor, nor association with any individual or group. Rather is was my early mastery of the Russian approach to the kinetic theory of plasmas that gave me an advantage. Different Russian and Western approaches to plasma theory developed in the period before the declassification of fusion research in 1956. In the West classification meant that only those who had the relevant security clearance could work on fusionrelated problems, and this did not include any theoretical astrophysicists. In contrast, in the Soviet Union, theoretical astrophysicists were intimately involved in the fusion program, and among their specific contributions was a knowledge of radiation transfer theory. As a result the Russians developed a treatment of emission and absorption processes in plasmas similar to conventional radiation transfer theory. The Russian approach incorporated the quantum mechanical concept of detailed balance, in the form of the Einstein coefficients. After declassification, this approach was viewed with considerable suspicion by Western plasma physicists, who had developed the theory in an entirely classical manner. There were several years of controversy in the plasma literature of the early 1960s before it became accepted in the West that the much more powerful Russian approach is formally equivalent to the Western approach. Nevertheless many still had reservations about it - a referee in a report on one of my papers told me that it is wrong to use a quantum mechanical formalism to treat a purely classical problem. This is nonsense: the modern-day theory of electrodynamics is Quantum Electrodynamics, based on the interaction of the electromagnetic and Dirac fields, and classical plasma physics should be regarded as an approximation to such a theory of Quantum Plasmadynamics. (I am currently nearing completion of a two-volume book with this general title.)

The important difference between the Russian and Western approaches can be understood by considering how one treats the change in the distribution of particles due to their emission and absorption of waves. Using the Russian approach, one applies energy and momentum conservation to a single particle when it emits a wave quantum with energy $\hbar \omega$ and momentum, $\hbar \mathbf{k}$, and then appeals to detailed balance to derive kinetic equations for the waves and the particles. A purely classical approach encounters a difficulty: a classical treatment of radiation on its own does not conserve energy and momentum. For radiation by a single particle in vacuo one introduces the radiation reaction force to rectify this deficiency, but this cannot be generalised in a simple way to treat radiation reaction in a plasma. The approach adopted in the West was to treat the back reaction on a distribution of particles using classical kinetic theory. Compared to the Russian approach this is much more cumbersome, and requires different specific calculations for each different kind of plasma wave. In the Russian approach, conservation of energy is built in at a microscopic level by appealing to quantum mechanical ideas. 